\title{
Evidence of high-field radio-frequency hot spots due to trapped vortices in niobium cavities
}

\author{
G. Ciovati ${ }^{1, *}$ and A. Gurevich ${ }^{2}$ \\ ${ }^{1}$ Thomas Jefferson National Accelerator Facility, Newport News, Virginia 23606, USA \\ ${ }^{2}$ Applied Superconductivity Center, National High Magnetic Field Laboratory, Florida State University, \\ Tallahassee, Florida 32310, USA \\ (Received 26 August 2008; published 16 December 2008)
}

\begin{abstract}
Superconducting radio-frequency (rf) cavities made of high-purity niobium exhibit strong anomalous rf losses starting at peak surface magnetic fields of about 90-100 $\mathrm{mT}$ in the gigahertz range. This phenomenon is referred to as " $Q$ drop." Temperature maps of the cavity surface have revealed the presence of "hot spots" in the high magnetic field region of the cavities. Several models have been proposed over the years to explain this phenomenon but there is still no experimental evidence on the mechanisms behind such hot spots. In this work we show that at least some of the hot spots are due to trapped vortices responsible for the anomalous losses. Here we report experiments in which a local thermal gradient was applied to the hot spot regions of a cavity in order to displace the vortices. Temperature maps measured before and after applying the thermal gradient unambiguously show that the hot spots do move and change their intensities, allowing us to determine changes in the hot spot positions and strengths and their effect on the cavity performance. Results on a large-grain niobium cavity clearly show a different distribution and in some cases a weakening of the intensity of the "hot spots," suggesting new ways of improving the cavity performance without additional material treatments.
\end{abstract}

\section{INTRODUCTION}

One of the most outstanding scientific issues related to superconducting rf (gigahertz range) cavities made of high-purity (residual resistivity ratio $>200$ ) bulk niobium is the occurrence of strong $\mathrm{rf}$ losses starting at a peak surface magnetic field $\left(B_{p}\right)$ of about $90-100 \mathrm{mT}$ (the socalled $Q$ drop). These anomalous losses manifest themselves in overheated regions ("hot spots") in the high magnetic field area of the cavity. Over the past decade, several models have been proposed to explain this phenomenon but none of them has been able to explain the totality of all the experimental data, nor point out decisive experiments, which would unambiguously prove a particular mechanism. An effective way of reducing the $Q$ drop is a low-temperature $\left(100-140^{\circ} \mathrm{C}\right)$ "in situ" baking of the cavity in ultrahigh vacuum for several hours [1]. Recent experimental work on niobium cavities provided clues for thermomagnetic breakdown, possibly initiated by magnetic vortices pushed into niobium by the rf field as a possible cause of the $Q$ drop [2]. The recent theoretical work has provided a good description of the $Q_{0}\left(B_{p}\right)$ curves (where $Q_{0}$ is the quality factor of the cavity's $\mathrm{TM}_{010}$ mode) by evaluating the contribution of regions of the cavity with reduced superconducting properties to the overall surface resistance [3].

*gciovati@jlab.org
Given that rf hot spots can be due to many mechanisms, such as local degradation of the surface resistance due to nonuniform impurity or oxide distributions, field enhancement by surface roughness, local field emission, or dissipation at grain boundaries (see, e.g., the review [1] and the references therein), distinguishing between these possibilities generally requires complex electromagnetic, chemical, and nanostructural characterizations of a particular hot spot. In that respect hot spots due to trapped vortices stand out and can be identified rather unambiguously because vortices (unlike structural defects) can be moved by comparatively weak disturbances like currents, ultrasound waves, or thermal gradients. So for cavities with no multipacting, any change of thermal maps after applying these disturbances would indicate that those hot spots, which change their position or intensity, are due to trapped vortices [4].

A possible way to reveal hot spots of magnetic vortices already trapped in the material is to depin them by applying a thermal gradient across and along the cavity wall [4]. In this contribution we present experimental results on the effects of applying a small local thermal gradient to the hot spots, identified on the cavity surface by temperature mapping, while the cavity is immersed in superfluid helium. The experiments were done on a large-grain and on a standard fine-grain niobium cavity.

The paper is organized as follows: a brief theoretical background for the experiments is given in Sec. II, and a 
description of the heater calibration is given in Sec. III. The results on the large-grain and fine-grain cavities are presented in Secs. IV and V, respectively. Section VI provides a discussion of the results and some conclusions.

\section{THEORETICAL BACKGROUND}

Magnetic vortices, resulting from the nonperfect shielding of the Earth's magnetic field or from thermoelectric currents induced during the cavity cooldown below the critical temperature, can be trapped at defects such as nonsuperconducting nanoprecipitates, grain boundaries, dislocations, and clusters of impurities [5]. In a recent article [6], the contribution of trapped vortices to the surface resistance in type-II superconductors has been calculated at both low and high rf fields and some important aspects can be summarized as follows: (i) There exists a vortex-free layer, of depth $d_{m}$ from the surface, in which the attraction force of vortices parallel to the surface is greater than the pinning force. (ii) The temperatureindependent contribution to the residual resistance due to trapped vortices, $R_{i}$, depends strongly on the position of the pinned vortices and on the size of the pinned vortex segments. $R_{i}$ is larger as the normal electrons' mean-free path decreases, while it is reduced by shorter pinned vortex segments. (iii) At high field, even the weak overheating due to oscillating trapped vortices is amplified and spread over areas much larger than the size of the source, due to the exponential dependence of the Bardeen-CooperSchrieffer (BCS) surface resistance on temperature. The field dependence of the hot spot's temperature and area depends on the detailed properties of the heat source and is different, for example, for sparse and overlapping hot spots.

A convenient way to try to eliminate vortices close to the rf surface is to apply a thermal force, $f_{T}=-s_{\phi} \nabla T$, greater than the pinning force. Here $s_{\phi}=\phi_{0}\left|\partial H_{c 1} / \partial T\right|$ is the vortex transport entropy per unit vortex length, $\phi_{0}$ is the magnetic flux quantum, and $H_{c 1}$ is the lower critical magnetic field [7]. The vortex depinning condition is achieved for a minimum thermal gradient $|\nabla T|_{c}$ which can be estimated from

$$
|\nabla T|_{c}=\frac{J_{c}}{\left|\partial H_{c 1} / \partial T\right|} \cong \frac{J_{c} \mu_{0} T_{c}^{2}}{2 B_{c 1} T}
$$

Taking $B_{c 1}(0 \mathrm{~K})=170 \mathrm{mT}$, the critical temperature $T_{c}=$ $9.25 \mathrm{~K}$ and a depinning critical current density $J_{c}=$ $1 \mathrm{kA} / \mathrm{cm}^{2}$ for niobium, we obtain $|\nabla T|_{c}=0.75 \mathrm{~K} / \mathrm{mm}$ at $4.2 \mathrm{~K}$. This corresponds to a temperature difference of about $2.3 \mathrm{~K}$ across the $3 \mathrm{~mm}$ thick cavity wall, which can be easily achieved on $\mathrm{Nb}$ cavities. Yet the critical thermal gradient increases greatly in materials such as $\mathrm{Nb}_{3} \mathrm{Sn}$ or $\mathrm{MgB}_{2}$ with larger $T_{c}$ and smaller $H_{c 1}$. For example, for $\mathrm{Nb}_{3}$ Sn with $T_{c}=18 \mathrm{~K}, H_{c 1}=38 \mathrm{mT}$, Eq. (1) yields $|\nabla T|_{c} \approx 27 \mathrm{~K} / \mathrm{mm}$ at $T=2 \mathrm{~K}$ for the same $J_{c}$ value used for $\mathrm{Nb}^{1}{ }^{1}$ In the absence of field emission or multipacting, changes of temperature maps after applying a small local heating could only be explained by the displacement of trapped vortices.

\section{HEATER CALIBRATION}

This section describes the measurements aimed at determining a proper heater configuration to provide the necessary temperature gradient across the cavity wall. The heater consists of a $100 \Omega, 1 / 8 \mathrm{~W}$ Allen-Bradley carbon resistor $(4 \mathrm{~mm} \times 2 \mathrm{~mm}$ area) encapsulated in a G10 block, to provide some isolation from the He bath. A pogo stick on the back side provides a spring loaded contact. The heater is built in the same way as the resistance temperature device (RTD) used in the thermometry system to map the temperature of the outer cavity surface [8]. In order to determine the amount of power necessary to produce the desired temperature gradient across the niobium wall, the heater is pushed in contact with the outer surface of a high-purity niobium disk, $120 \mathrm{~mm}$ diameter, $3 \mathrm{~mm}$ thick. The disk is sealed to a stainless steel can with indium wire. Seven Allen-Bradley RTDs, about $1 \mathrm{~cm}$ spaced, are in contact with the inner surface of the $\mathrm{Nb}$ disk, centered with respect to the heater. Apiezon $\mathrm{N}$ grease is used to assure a good thermal contact between the AllenBradley resistors and the $\mathrm{Nb}$ surface. Figure 1 shows a schematic of the experimental setup.

The can is evacuated to about $10^{-5}$ mbar and is immersed in liquid helium at $4.3 \mathrm{~K}$. The temperature is subsequently lowered to $2.0 \mathrm{~K}$ by pumping on the $\mathrm{He}$ bath. The $R(T)$ of the RTDs is measured between 10 and $2.0 \mathrm{~K}(10 \mu \mathrm{A}$ excitation current). Two calibrated silicon diodes, one in contact with the inner surface of the $\mathrm{Nb}$ disk, one in contact with the liquid helium, are used as reference. The heater current is increased and the temperature distribution on the inner surface of the $\mathrm{Nb}$ disk is measured in steady state as a function of the heater power, $P$, at fixed $\mathrm{He}$ bath temperatures, $T_{b}(4.3$ and $2.0 \mathrm{~K})$. At high enough power levels, film boiling in He I can occur, introducing a hysteretic behavior in the $P(T)$ dependence. Since the onset of film boiling decreases significantly as the orientation of the heated surface changes from upward to downward [9], we did the experiment with the disk oriented downward and at $90^{\circ}$ between the normal to the surface and the vertical direction. Plots of the temperature difference between the hottest point on the $\mathrm{Nb}$ inner surface and the He bath, $\Delta T_{\max }$, as a function of the heater power at $4.3 \mathrm{~K}$ and $2.0 \mathrm{~K}$ are shown in Fig. 2 for the two orientations. As expected, the temperature gradient is much more sensitive to the surface orientation in $\mathrm{He} \mathrm{I}$ at $4.3 \mathrm{~K}$, than in $\mathrm{He}$

\footnotetext{
${ }^{1}$ The calculated value of $|\nabla T|_{c}$ for $\mathrm{Nb}_{3} \mathrm{Sn}$ will be actually even larger when considering the much higher $J_{c}$ than $\mathrm{Nb}$ for this material.
} 


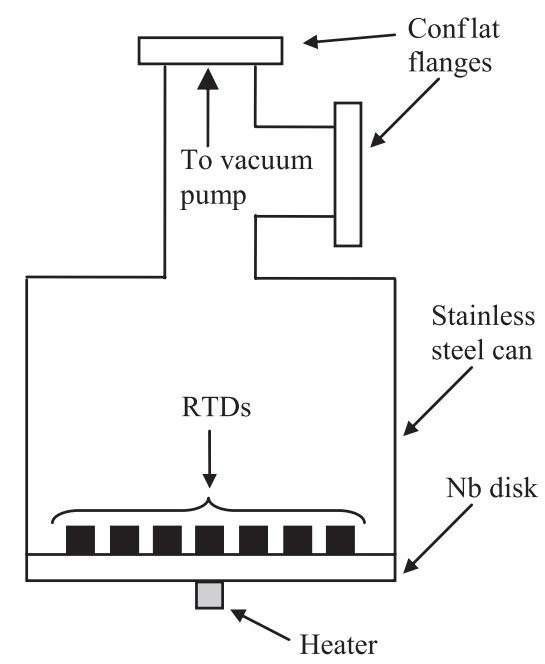

FIG. 1. Schematic representation of the test setup for the heater calibration.

II at $2.0 \mathrm{~K}$. Since the orientation of the cavity surface changes from $\pm 30^{\circ}$ to $\pm 150^{\circ}$ along the contour, near the equator, we decided to operate the heaters at a bath temperature of $2.0 \mathrm{~K}$ and at a power of about $6 \mathrm{~W}(300 \mathrm{~mA}$ excitation current), corresponding to a temperature gradient between the inner $\mathrm{Nb}$ surface and the bath of about $3 \mathrm{~K}$. Figure 3 shows the normalized spatial temperature distribution on the inner surface of the $\mathrm{Nb}$ disk for different values of heater power, measured at $2.0 \mathrm{~K}$ and at a surface orientation of $90^{\circ}$. The full-width half-maximum at $6 \mathrm{~W}$ is about $13 \mathrm{~mm}$. With an excitation current of $300 \mathrm{~mA}$, the heater body warms up significantly, as its resistance drops from about $7 \mathrm{k} \Omega$ to about $66 \Omega$, a value lower than the one measured at $300 \mathrm{~K}$. The obtainable power saturates above

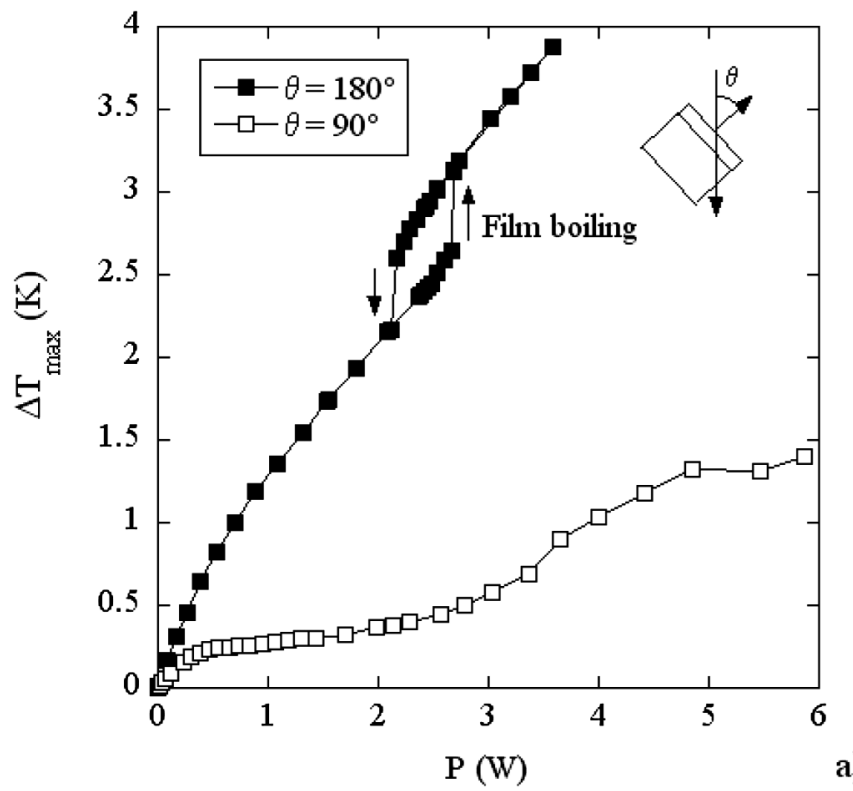

$6 \mathrm{~W}$, as an increase in the excitation current is compensated by a decrease of resistance, due to self-heating.

In order to estimate the effective temperature gradient across the $\mathrm{Nb}$ wall as a function of the distance from the heater location, we did a thermal analysis of a $3 \mathrm{~mm}$ thick disk with the heater in the center using the finite element code ANSYS [10]. Shown in Fig. 4 are the thermal conductivity, $\kappa(T)$, measured on a high-purity $\mathrm{Nb}$ sample, and Kapitza conductance, $h(T)$, as a function of temperature typically reported for chemically polished $\mathrm{Nb}$ used in the simulation. For surface temperatures $T_{s}$ above the $\lambda$ point $(2.17 \mathrm{~K})$, we used a constant heat transfer coefficient of $1 \mathrm{~kW} / \mathrm{m}^{2} \mathrm{~K}$, as indicated in [11] for $T_{s}$ up to $25 \mathrm{~K}$. The value of the heater's heat flux into the $\mathrm{Nb}$ surface is used as a parameter in the simulation to fit the measured temperature distribution on the inner surface. This value will be lower than the heater's dissipated power, because of some cooling from the He bath and of reduced heat transfer efficiency through the Apiezon grease layer and through interfaces. The calculation shows that only about $30 \%$ of the heater's power is transmitted to the outer $\mathrm{Nb}$ surface. Figure 5 shows the temperature gradient, across the $\mathrm{Nb}$ wall, as a function of the lateral distance from the heater, for a heater power of $6 \mathrm{~W}$. The obtained value is close to the minimum required to depin vortices, as estimated with Eq. (1). The intensity of the thermal force is significant only within the heater area and it is directed inward. This is not the optimal situation, since vortices will be pushed towards the rf surface, unless they fall within the vortexfree layer. On the other hand, it is not feasible to install the heaters, or any other object, on the inner $\mathrm{Nb}$ surface since it will prevent achieving high rf fields inside the cavity, because of the low quality factor.

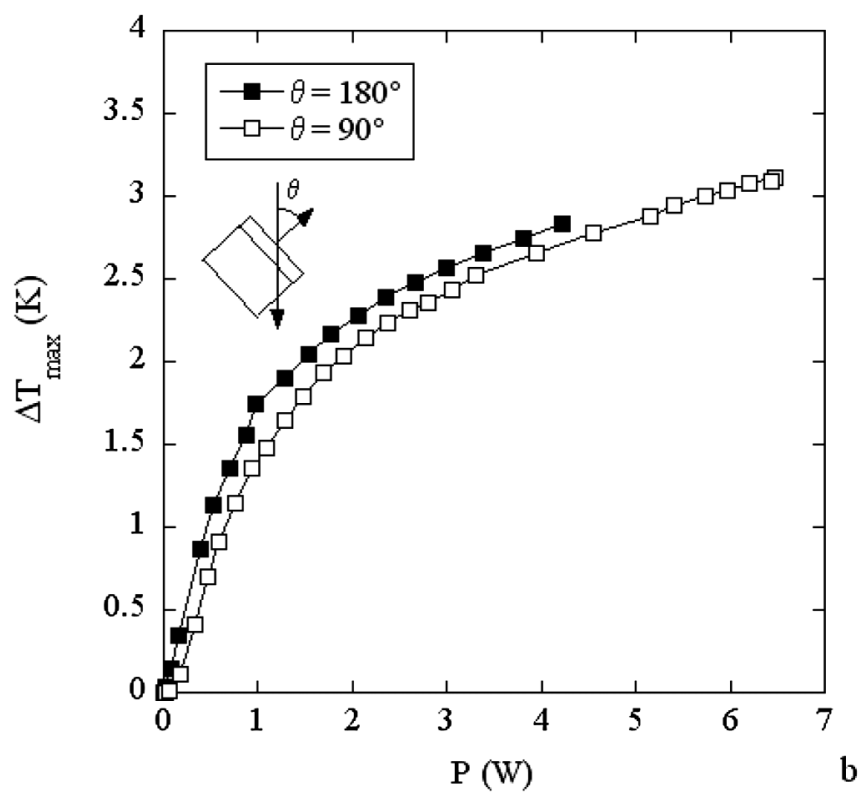

FIG. 2. Maximum temperature difference between the value at the inner surface of the Nb disk and the He bath as a function of the heater power for two orientations of the disk, measured at $4.3 \mathrm{~K}$ (a) and $2.0 \mathrm{~K}$ (b). 


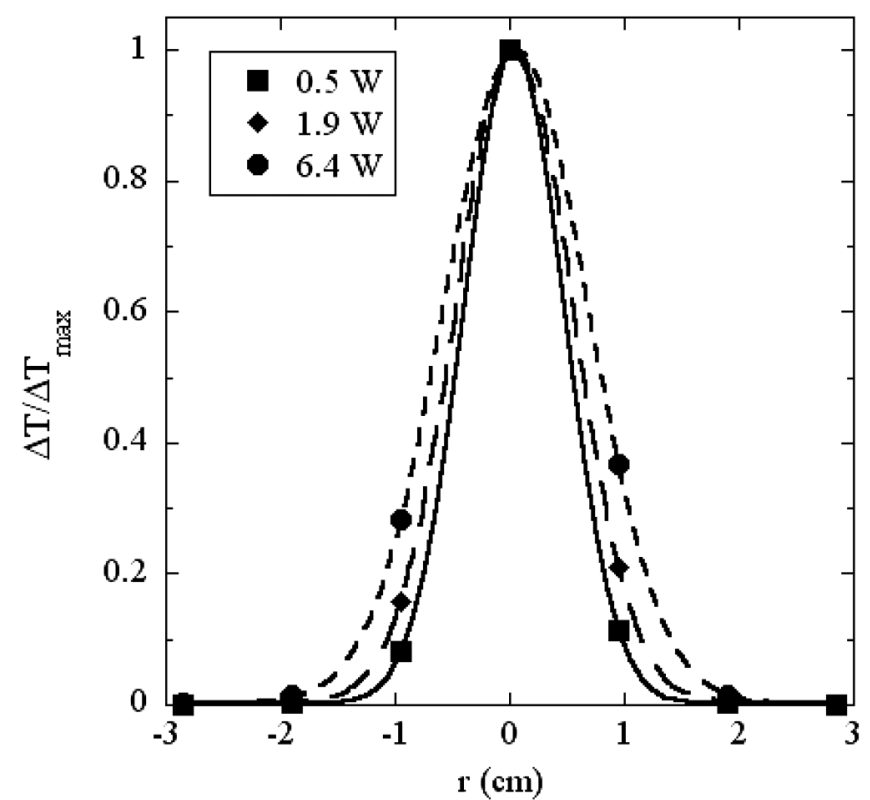

FIG. 3. Normalized temperature gradient between the inner surface of the $\mathrm{Nb}$ disk and the $\mathrm{He}$ bath temperature as a function of the distance from the center of the disk for different values of heater power, measured at $2.0 \mathrm{~K}$, surface oriented at $90^{\circ}$. The lines are Gaussian fits to the data.

\section{LARGE-GRAIN CAVITY TEST RESULTS}

The cavity used for this experimental study has the same shape as the one used in the CEBAF accelerator [12]. The cavity, built from a large-grain high-purity ingot made by Oriental Tantalum and Niobium Company, Ningxia,

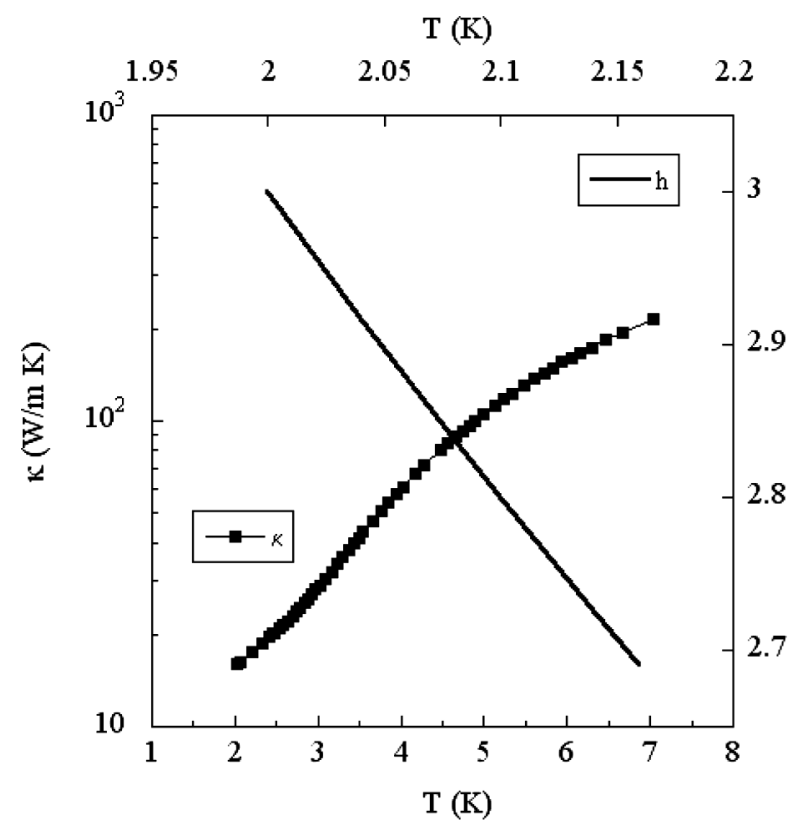

FIG. 4. Thermal conductivity of $\mathrm{Nb} k$ and heat transfer coefficient $h$ used in the thermal analysis.

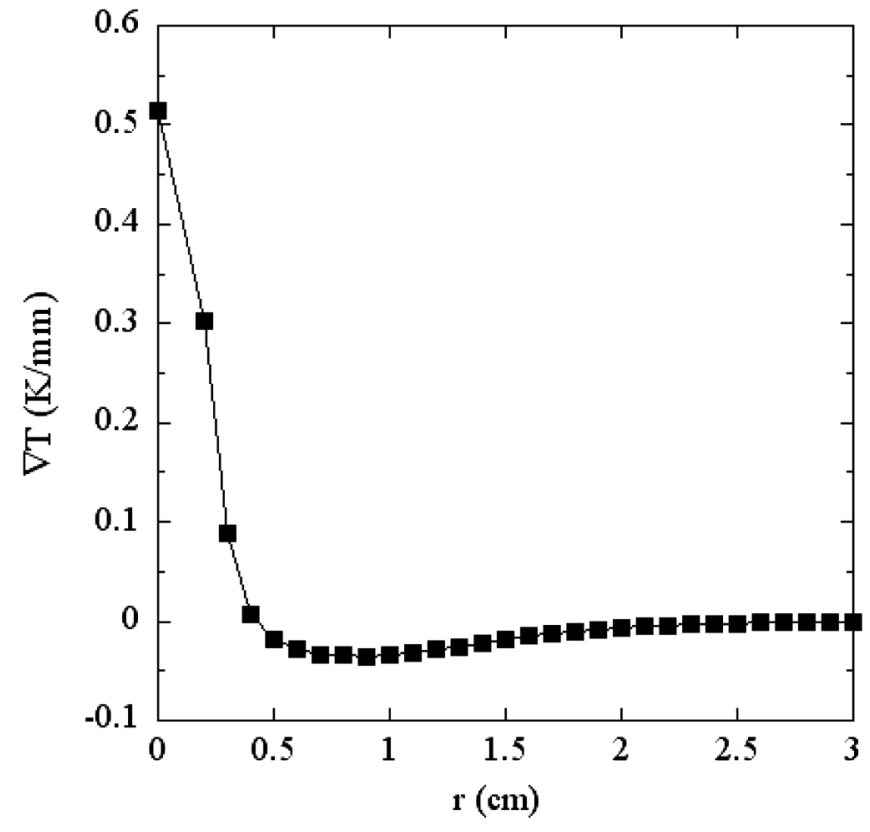

FIG. 5. Temperature gradient across the $\mathrm{Nb}$ wall as a function of the lateral distance from the heater (at $r=0$ ), for a nominal heater power of $6 \mathrm{~W}, T_{b}=2.0 \mathrm{~K}$, calculated with ANSYS.

China, had been previously used for a study published in [2]. The polishing method of the rf surface is buffered chemical polishing. The standard cavity preparation, thermometry system assembly and calibration steps were reported in detail in [2].

\section{A. Test no. 1 results}

The $Q_{0}\left(B_{p}\right)$ curve measured at $2 \mathrm{~K}$ for the first baseline test is shown in Fig. 6. The cavity exhibits the $Q$ drop starting at about $100 \mathrm{mT}$, with no field emission. The temperature map at $118 \mathrm{mT}$, shown in Fig. 7(a), indicates

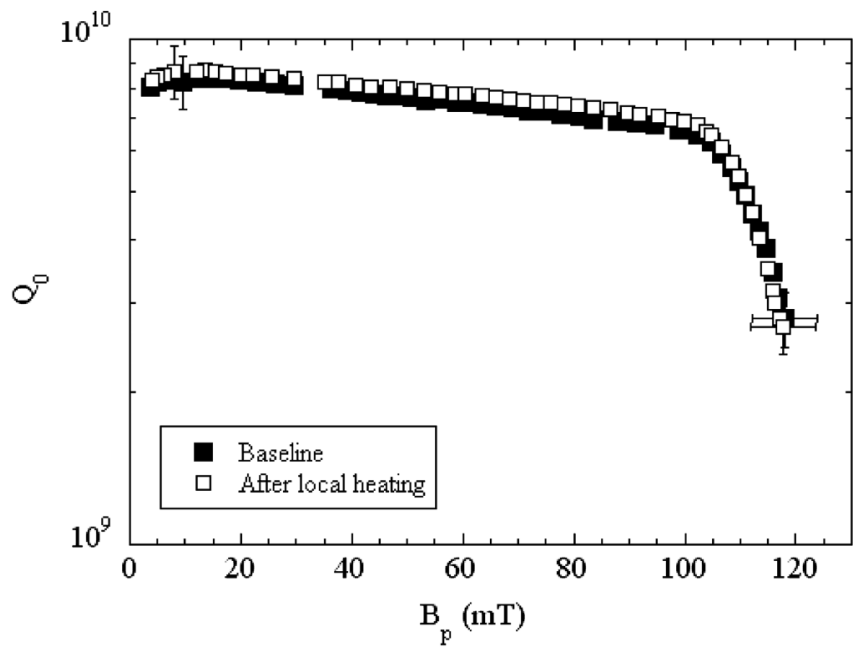

FIG. 6. $Q_{0}\left(B_{p}\right)$ measured at $2 \mathrm{~K}$ before and after applying local heating at some hot spot locations. 

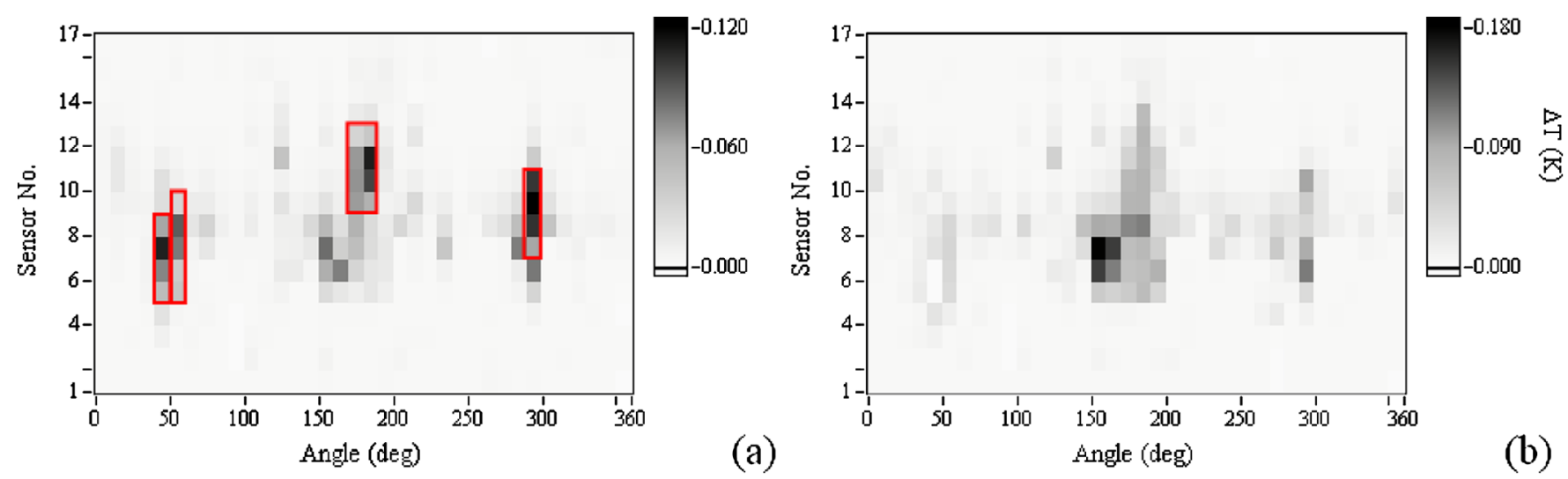

FIG. 7. (Color) "Unfolded" temperature maps of the large-grain cavity at $B_{p}=118 \mathrm{mT}, T_{b}=2 \mathrm{~K}$ before (a) and after (b) applying local heating in the regions enclosed in the red boxes shown in (a). The upper iris of the cavity corresponds to sensors no. 1 and the lower iris to sensors no. 16. There are no thermometers directly on the equator weld but immediately below (sensors no. 9) and above (sensors no. 8). Note the different $\Delta T$ scales. In the equator region, the distance between adjacent thermometers in the azimuthal direction is approximately twice as large as the distance along the longitudinal direction.

few hot spots in the equator area. After the first round of $Q_{0}\left(B_{p}\right)$ measurements at $2 \mathrm{~K}$, the rf power was shut off and the thermometers on some hot spot locations [highlighted with a red box in Fig. $7(\mathrm{a})^{2}$ ] were used as heaters by providing a sequential excitation current of $300 \mathrm{~mA}$, for about $10 \mathrm{~s}$ each. The cryostat was refilled with liquid $\mathrm{He}$ at $4.3 \mathrm{~K}$ and all the thermometers were recalibrated between 4.3 and $2 \mathrm{~K}$. The subsequent high-power rf test still showed $Q$ drop but the thermometers which were used as heaters showed very little heating compared to the baseline test. We suspected that the viscosity of the Apiezon grease layer between the thermometers and the cavity was reduced by the hot body of the thermometer during heating and allowed some superfluid He to flow between the surface of the resistor and the cavity surface, effectively reducing its sensitivity. Therefore we warmed up the cavity to $300 \mathrm{~K}$ and applied a new layer of grease on the heated thermometers. A new $Q_{0}\left(B_{p}\right)$ measurement at $2 \mathrm{~K}$ and the temperature map at $118 \mathrm{mT}$, shown in Figs. 6 and 7(b), respectively, revealed reduced $\mathrm{rf}$ losses at some of the heated locations, while higher losses were detected in other areas. Figure 8 shows the temperature difference between the cavity surface and the He bath, $\Delta T$, as a function of the applied $\mathrm{rf}$ field for some thermometers before and after heating (with a new layer of grease applied). The $\Delta T\left(B_{p}\right)$ dependence can be fitted with a power law $\Delta T=A B_{p}{ }^{n}$, with $n>20$, practically indistinguishable from the exponential field dependence. The reduction of residual resistance, obtained from a fit of $R_{S}(T)$ data, after heating is within the experimental error (from $12.9 \pm 1.2$ to $12.2 \pm 1.3 \mathrm{n} \Omega$ ).

\footnotetext{
${ }^{2}$ The heat distribution around a hot spot is fairly uniform. The temperature map in Fig. 7(a) may give the impression of "line heating" because the spacing between adjacent thermometers in the azimuthal direction is approximately twice as large as the distance along the longitudinal direction.
}

Although it is convenient to use the same resistors both as thermometers and as heaters, their sensitivity and resolution ( $d R / d T$ decreased by about $30 \%$ after heating) is reduced after heating, making the data less reliable. Therefore we decided to use independent resistors glued to the cavity surface to be used as heaters in the next tests.

\section{B. Test no. 2 results}

The cavity was kept under vacuum after test no. 1 and seven heaters were glued in the space between two neighboring thermometry boards in the equator regions near angles $150^{\circ}$ and $290^{\circ}$. The heaters were glued using an electrical insulating, thermally conductive epoxy adhesive (DeltaBond $^{\mathrm{TM}} 155$ ). The results of the rf test at $2 \mathrm{~K}$ are

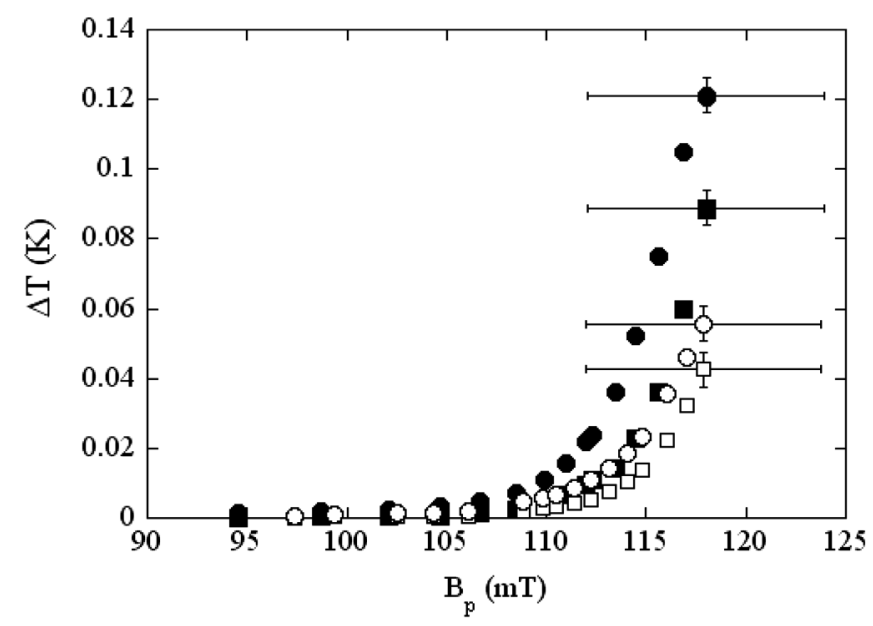

FIG. 8. Temperature increase above the He bath at $2 \mathrm{~K}$ as a function of $B_{p}$ for sensors 50-8 (squares) and 290-9 (circles) before (solid symbols) and after (empty symbols) they were used as heaters and a new layer of grease was applied. The sensors are identified by the pair angle-sensor numbers. 


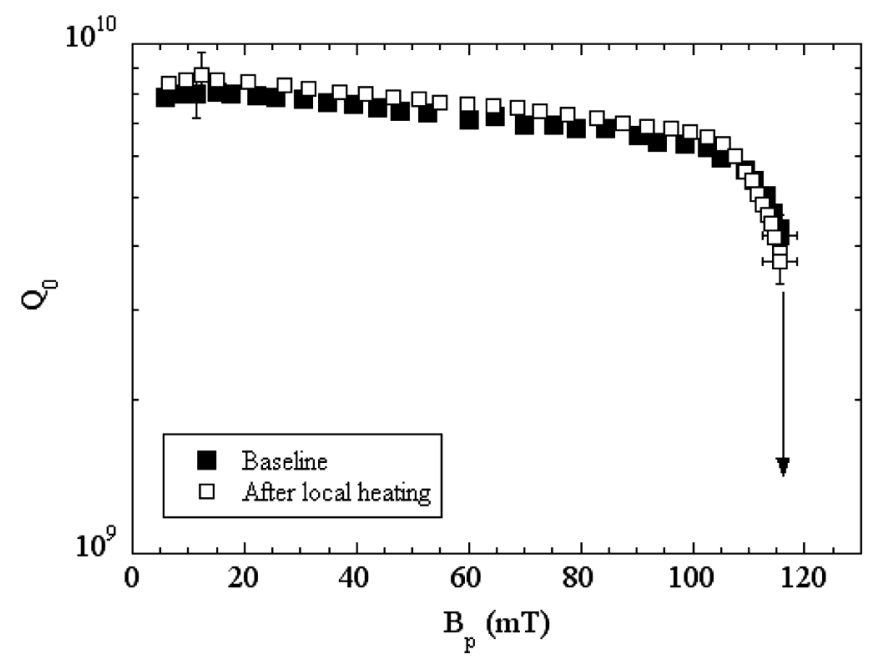

FIG. 9. $Q_{0}\left(B_{p}\right)$ measured at $2 \mathrm{~K}$ during the second test, before and after applying local heating at some hot spot locations. The quench at $116 \mathrm{mT}$ is due to a reduced heat transfer to the He bath caused by five heaters glued near the location 150-7.

shown in Fig. 9: the performance was limited by $Q$ drop, without field emission, and quench near the sensor 150-7 (the first number is the angle location, the second one is the sensor number), due to the limited cooling by the superfluid $\mathrm{He}$ because of the heaters glued in that area. The temperature map of the cavity at $B_{p}=115 \mathrm{mT}$ is shown in Fig. 10, where the red dots indicate the heater location.

After the first high-power rise, the rf power is shut off and the heaters are sequentially powered up to $\sim 6 \mathrm{~W}$ for about $30 \mathrm{~s}$ each. After this operation, the $\mathrm{rf}$ power is switched on and a new $Q_{0}\left(B_{p}\right)$ curve is measured (shown in Fig. 9): changes of the quality factor are of the order of the experimental error $(10 \%)$. Nevertheless, a reduction in the hot spot intensity at $\sim 150^{\circ}$ was observed: this can be clearly seen in Fig. 11 , showing the $\Delta T(115 \mathrm{mT})$ at $150^{\circ}$ before and after heating. $\Delta T\left(B_{p}\right)$ for sensors 160-6 and 290-8 is plotted in Fig. 12, showing reduced losses in one
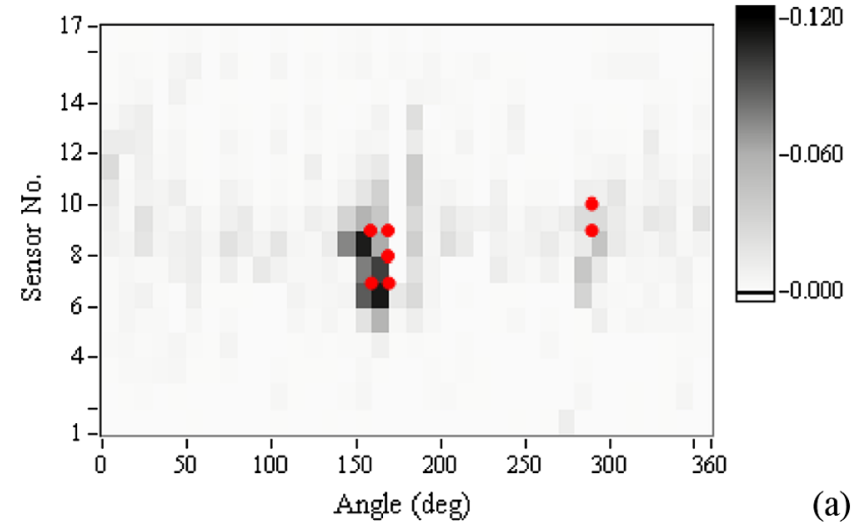

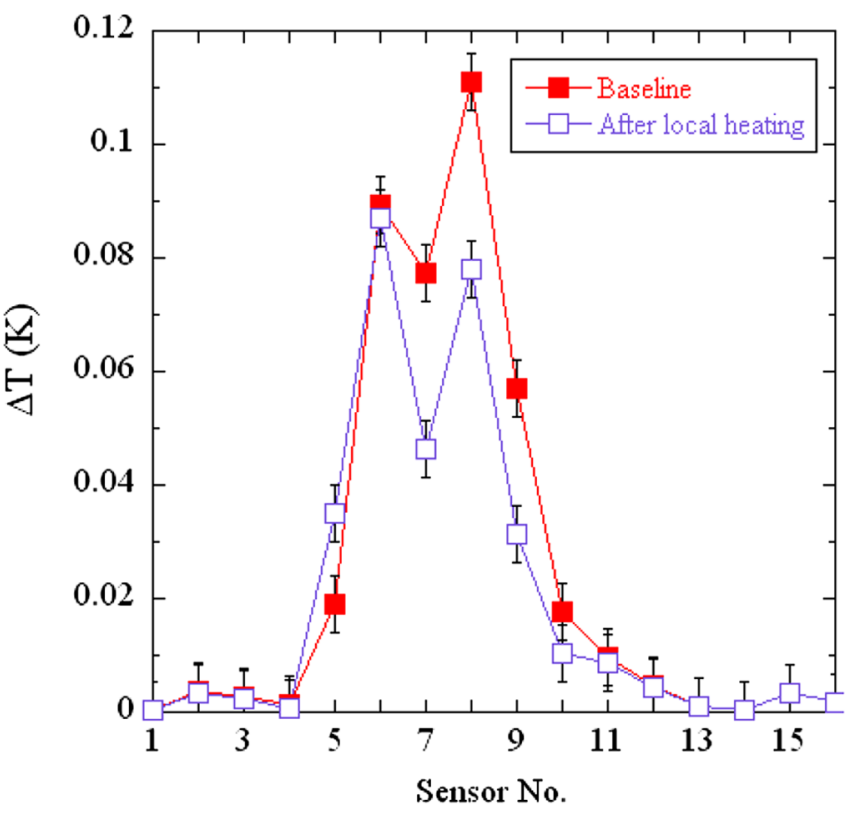

FIG. 11. (Color) $\Delta T(115 \mathrm{mT})$ above $2 \mathrm{~K}$, at $150^{\circ}$ from top (sensor no. 1) to bottom iris (sensor no. 16) of the cavity before and after applying local heating near sensors no. 7 and 9.

case (160-6) and no significant changes in the other (290$8)$.

\section{Test no. 3 results}

After test no. 2, about $20 \mu \mathrm{m}$ were removed from the inner cavity surface by BCP. The He bath temperature was lowered to $1.7 \mathrm{~K}$ in test no. 3 to take advantage of the higher resolution $d R / d T$ of the RTDs at lower temperature (from $\sim 7$ to $\sim 15 \Omega / \mathrm{mK}$ ). In the preliminary rf test result, the cavity performance was limited by $Q$ drop, without field emission, and the main hot spot location was in the equator region at $150^{\circ}$, as in the previous test. Five heaters were attached in that area and a new baseline was obtained after cool-down to $1.7 \mathrm{~K}$. The $Q_{0}\left(B_{p}\right)$ curve is shown in

FIG. 10. (Color) Unfolded temperature maps at $B_{p}=115 \mathrm{mT}, T_{b}=2 \mathrm{~K}$ before (a) and after (b) applying local heating using seven heaters attached to the cavity at the locations indicated by the red dots in (a). Note the different $\Delta T$ scales. 


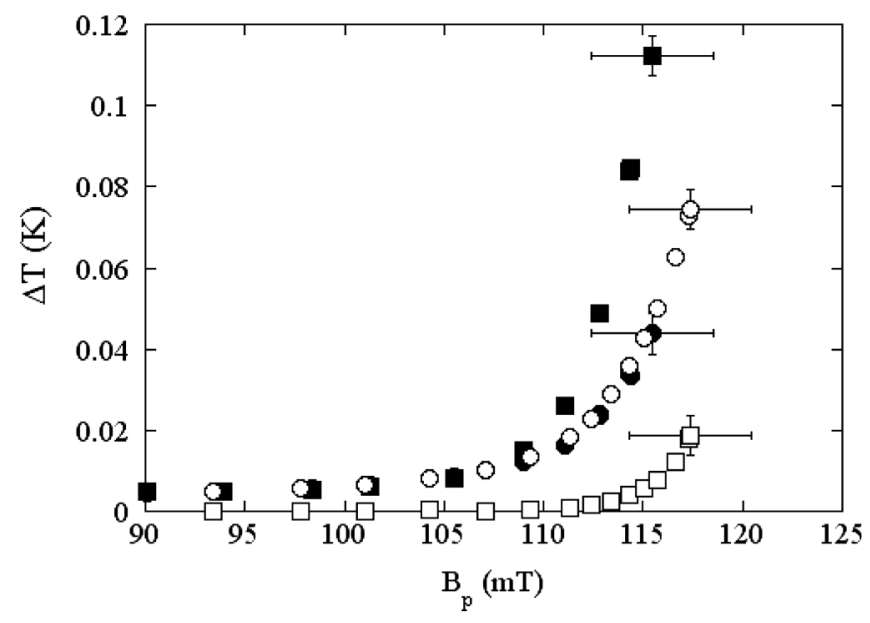

FIG. 12. $\Delta T\left(B_{p}\right)$ above the He bath at $2 \mathrm{~K}$ for sensors 160-6 (squares) and 290-8 (circles) before (solid symbols) and after (empty symbols) nearby heaters were powered up.

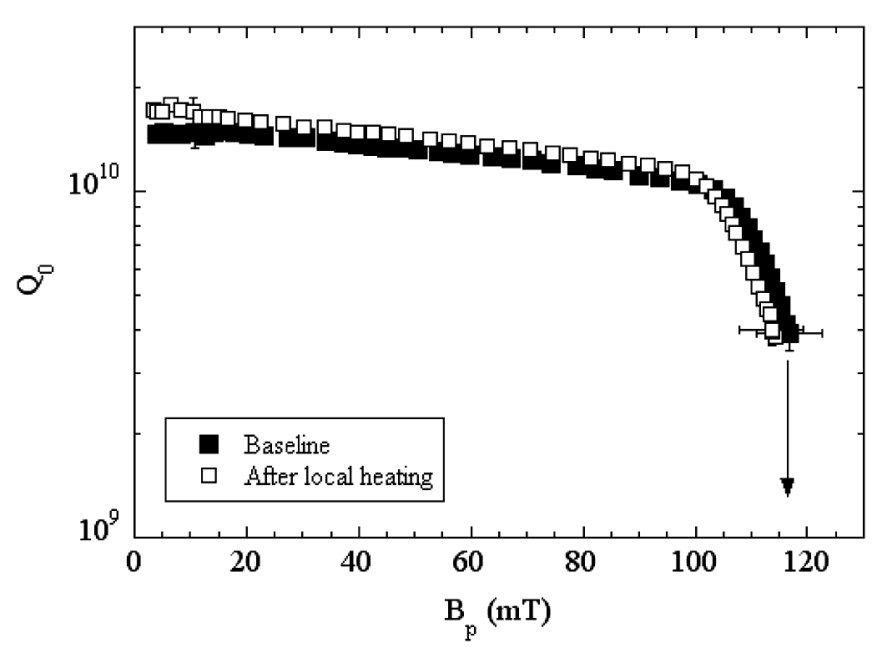

FIG. 13. $Q_{0}\left(B_{p}\right)$ measured at $1.7 \mathrm{~K}$ during the third test, before and after applying local heating at some hot spot locations.

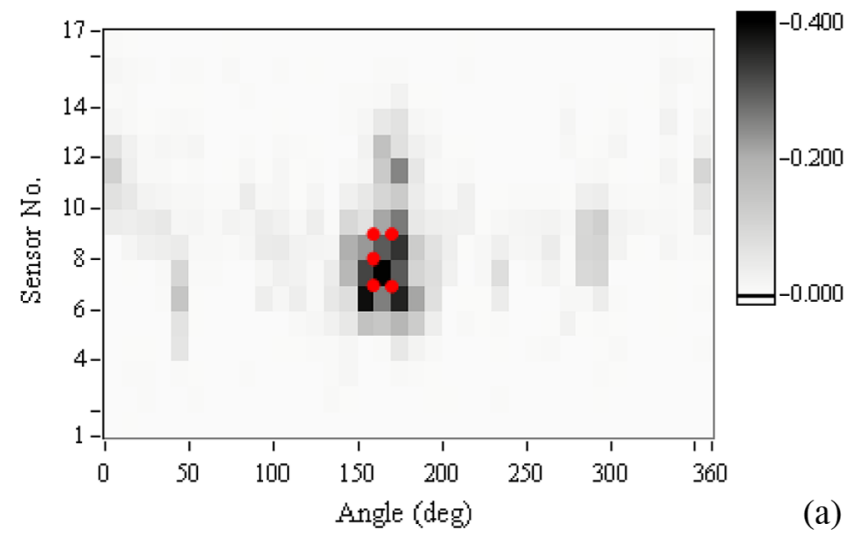

Fig. 13 and there were no significant changes neither in the cavity performance nor in the temperature maps by cycling between 300 and $1.7 \mathrm{~K}$. A new $Q_{0}\left(B_{p}\right)$ curve was measured after sequentially powering up the heaters to $6 \mathrm{~W}$, for $\sim 30 \mathrm{~s}$ each and the results are shown in Fig. 13: the residual resistance, obtained by fitting the $R_{s}(T)$ data at low field, decreased by about $15 \%$ from the initial value of $\sim 14 \mathrm{n} \Omega$ before heating, but no significant changes were obtained at high field. Temperature maps at $112 \mathrm{mT}$ before and after heating, shown in Fig. 14, indicate reduced losses in the region where the heaters were located, after the heaters were powered up. To show this even more clearly, a plot of $\Delta T(112 \mathrm{mT})$ at $160^{\circ}$ is shown in Fig. 15, before and after heating.

After test no. 3, we did an optical inspection of the inner cavity surface in the equator region at $150^{\circ}-170^{\circ}$, where high losses occurred, using a Questar telescope. The only noticeable features were grain boundaries near the equator weld, as shown in Fig. 16.

\section{FINE-GRAIN CAVITY TEST RESULTS}

The rf measurements on cavities made of fine-grain (ASTM 6, $50 \mu \mathrm{m}$ average grain size) high-purity niobium treated by BCP show very little improvement of the $Q$ drop by low-temperature baking [13]. We decided to try the local heating technique on this type of material/treatment combination to observe whether changes of the hot spot intensity and location occurred in a similar way as in the large-grain cavity. The cavity for this test has the same shape as the one used in the CEBAF accelerator and was built from high-purity, fine-grain $\mathrm{Nb}$ sheets from Wah Chang. The cavity was prepared for the rf test in the same way as the large-grain one (BCP treatment). The results of the preliminary high-power rf test at $1.72 \mathrm{~K}$ showed the $Q$ drop, without field emission, starting at about $80 \mathrm{mT}$. The cavity was warmed up to $300 \mathrm{~K}$ and seven heaters were glued at two hot spot locations. Both the

FIG. 14. (Color) Unfolded temperature maps at $B_{p}=112 \mathrm{mT}, T_{b}=1.7 \mathrm{~K}$ before (a) and after (b) applying local heating using five heaters attached to the cavity at the locations indicated by the red dots in (a). 


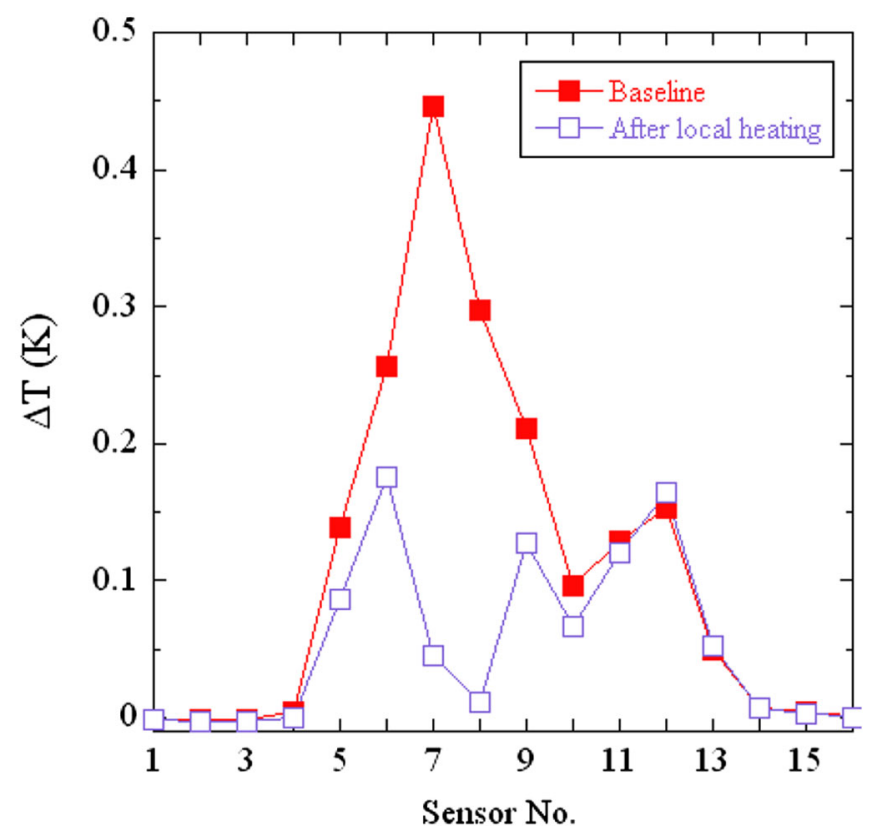

FIG. 15. (Color) $\Delta T(115 \mathrm{mT})$ above $1.7 \mathrm{~K}$, at $160^{\circ}$ from top (sensor no. 1) to bottom iris (sensor no. 16) of the cavity before and after applying local heating near sensors no. 7, 8, and 9. The error of the $\Delta T$ values is about $5 \mathrm{mK}$.

following baseline rf test at $1.72 \mathrm{~K}$, shown in Fig. 17, and the temperature map at $B_{p}=100 \mathrm{mT}$, shown in Fig. 18(a), confirmed the results of the preliminary test. The cavity quenched at $\sim 103 \mathrm{mT}$ due to the reduced heat transfer caused by the additional heaters attached to the cavity. The heaters were sequentially powered up to $\sim 6 \mathrm{~W}$, with no rf field in the cavity, and the $Q_{0}\left(B_{p}\right)$ curve was remeasured afterwards. The results, shown in Fig. 17, indicate an increase of the residual resistance (lower $Q_{0}$ at low field) by $7 \%$ from the initial value of about $4 \mathrm{n} \Omega$, and there was no significant change at high field. Interestingly, there was

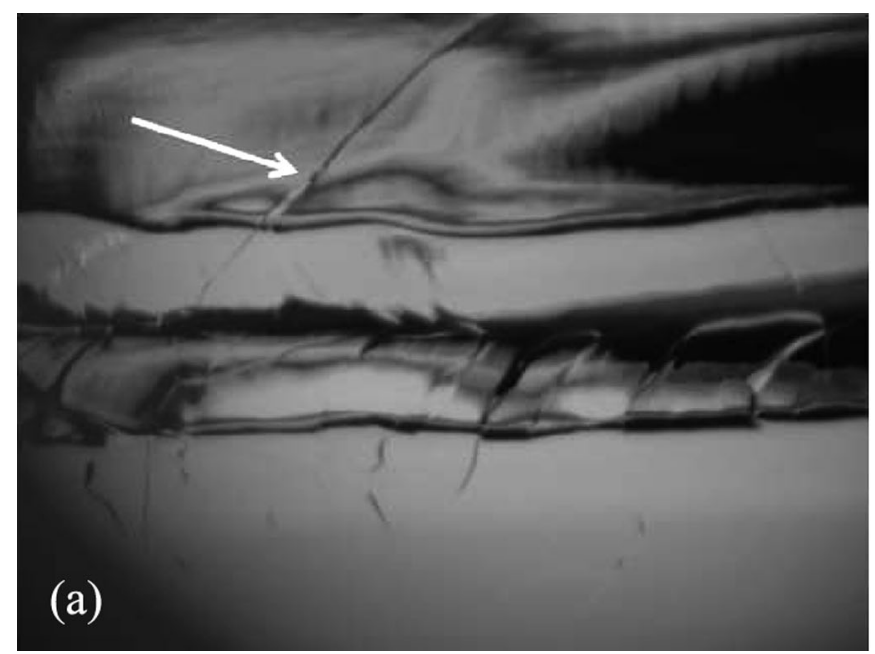

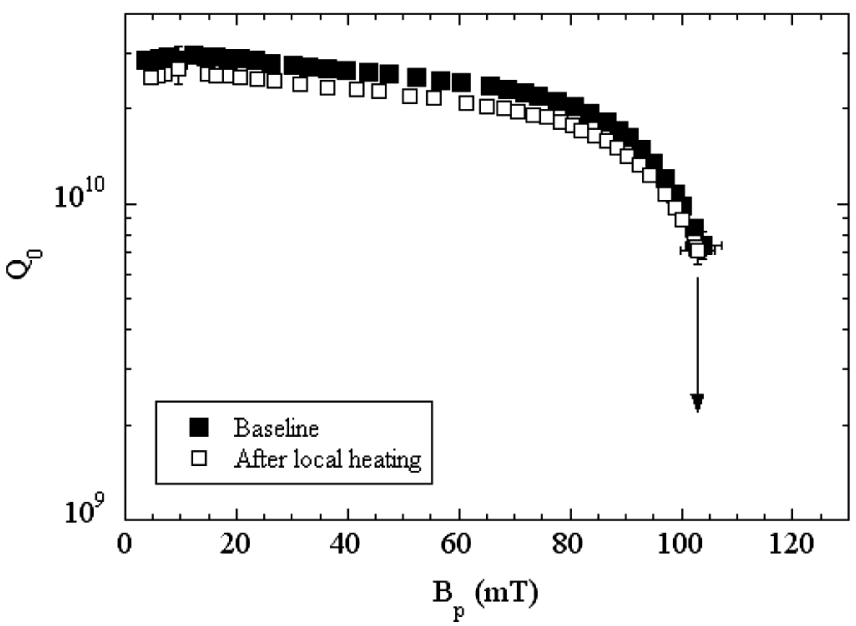

FIG. 17. $Q_{0}\left(B_{p}\right)$ measured on a fine-grain cavity at $1.72 \mathrm{~K}$, before and after applying local heating at some hot spot locations.

also no significant change in the temperature maps, as shown in Fig. 18(b) and 19.

An optical inspection of the inner cavity surface in the hot spot locations showed no outstanding feature. Nevertheless, the surface appears to be rougher, with a matte finish, compared to the surface of the large-grain cavity, as shown in Fig. 20: the appearance of the fine-grain structure is enhanced by the differential etching of the grains during the $\mathrm{BCP}$ process.

\section{DISCUSSION AND CONCLUSIONS}

The experimental results on a BCP-treated large-grain niobium cavity reported in Sec. IV clearly show a redistribution of hot spots and, in some cases, a reduction of $\mathrm{rf}$ losses by applying a local thermal gradient of about $0.5 \mathrm{~K} / \mathrm{mm}$ across the $\mathrm{Nb}$ wall. These results could only

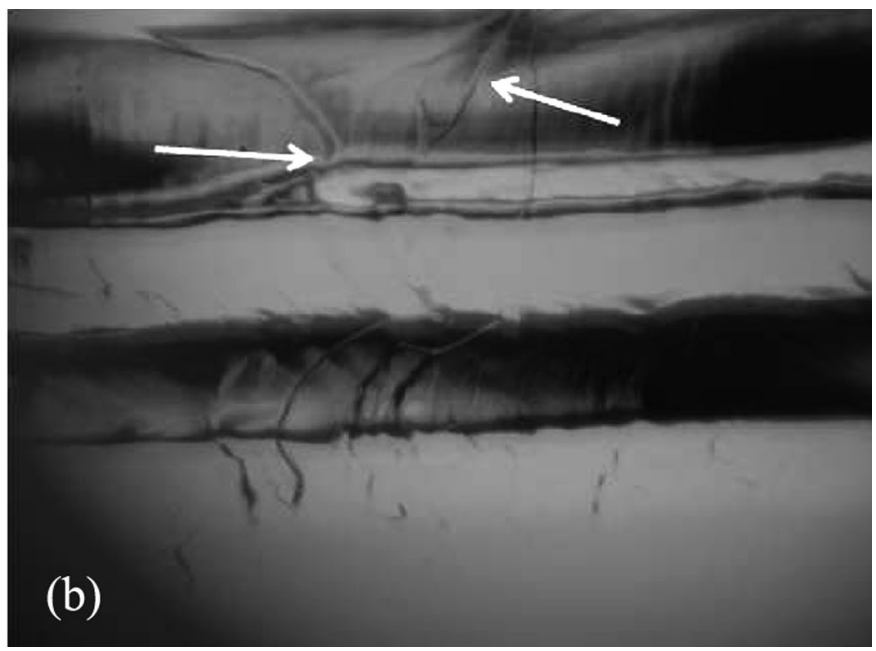

FIG. 16. Images of the inner cavity surface in the equator area at $150^{\circ}-170^{\circ}$ where high losses were found by temperature mapping. Grain boundaries (indicated by the white arrows) are well visible. As a reference scale, the width of the weld is $\sim 4 \mathrm{~mm}$. 

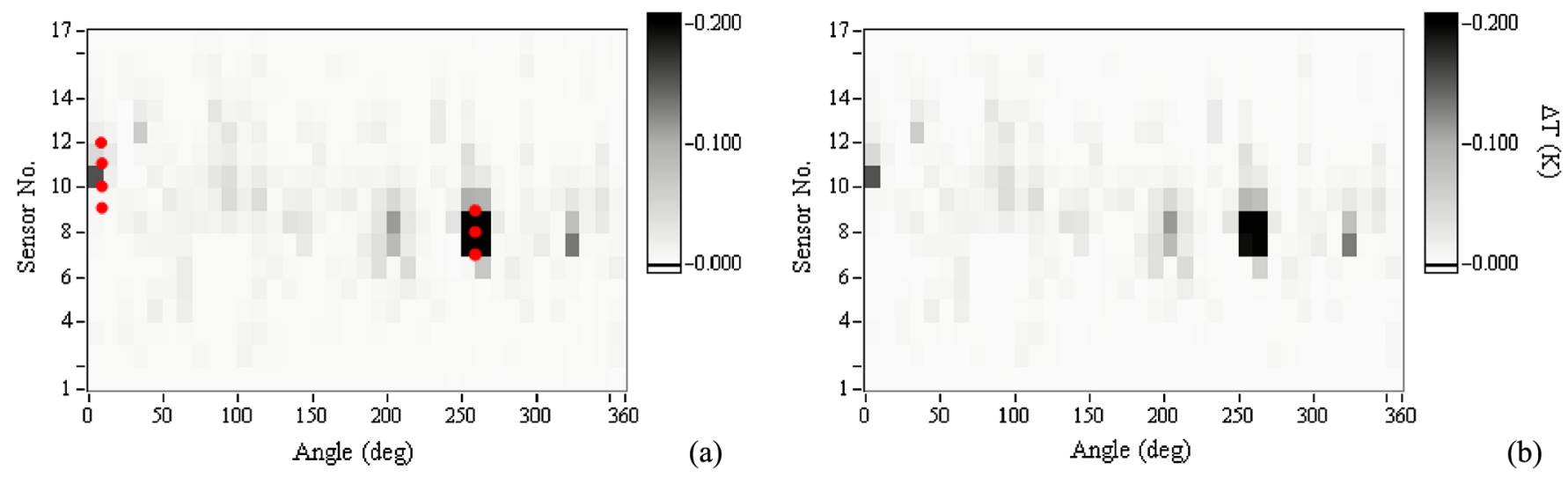

FIG. 18. (Color) Unfolded temperature maps at $B_{p}=100 \mathrm{mT}, T_{b}=1.72 \mathrm{~K}$ before (a) and after (b) applying local heating using seven heaters attached to the cavity at the locations indicated by the red dots in (a).

be explained by depinning of magnetic vortices by the thermal force, revealing the underlying mechanism behind some of the hot spots and suggesting a simple way of improving the cavity performance based on developing more effective ways of eliminating trapped vortices after the cavity cooldown. The presence of additional hot spots due to field cooling in a large fraction of the Earth's magnetic field and how material treatments can affect the trapping efficiency had been demonstrated already in [14].

In our experiments the Earth's magnetic field was shielded to about $2 \mathrm{mG}$, which corresponds to a density of about $10^{4}$ vortices per $\mathrm{cm}^{2}$ in the case of complete flux penetration at $T=T_{c}$. Vortices induced by either the residual Earth field or transient circulating thermoelectric

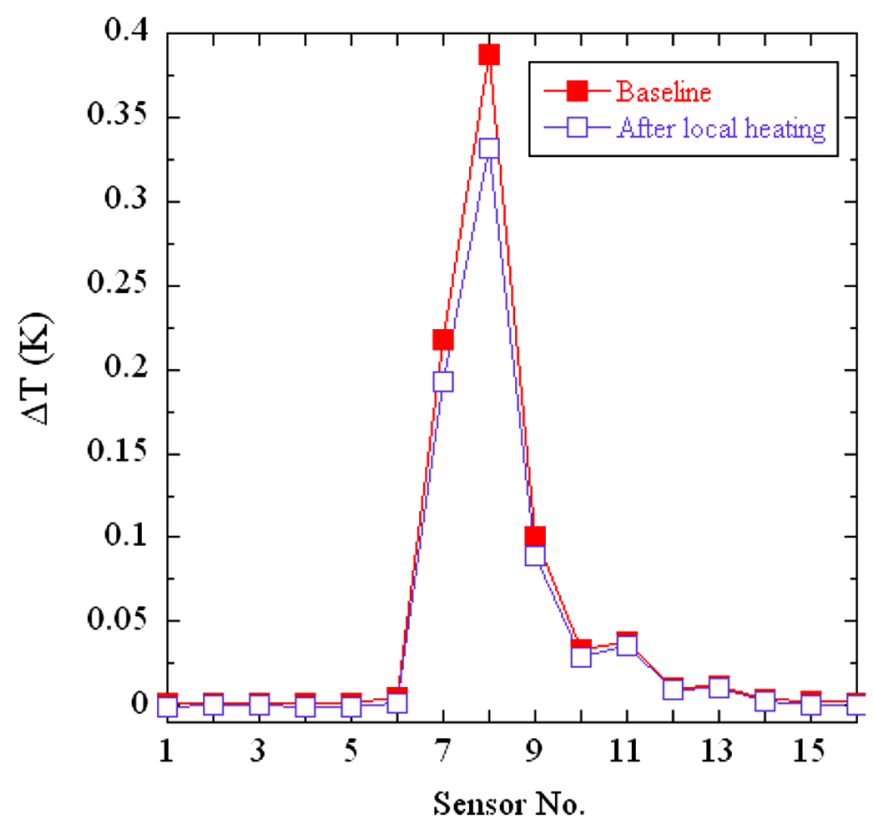

FIG. 19. (Color) $\Delta T(100 \mathrm{mT})$ above $1.72 \mathrm{~K}$, at $250^{\circ}$ from top (sensor no. 1) to bottom iris (sensor no. 16) of the fine-grain cavity before and after applying local heating near sensors no. 7 , 8 , and 9 . The error of the $\Delta T$ values is about $5 \mathrm{mK}$. currents during the cavity cooldown below $T_{c}$ can be distributed very inhomogeneously, particularly in the high purity, cavity grade $\mathrm{Nb}$ in which pinning of vortices is very weak as compared to technical superconductors (typical critical current densities $J_{c}=0.1-1 \mathrm{kA} / \mathrm{cm}^{2}$ in $\mathrm{Nb}$ are 3-4 orders of magnitude lower than $J_{c}$ of $\mathrm{NbTi}$ or $\mathrm{Nb}_{3} \mathrm{Sn}$ used in wires of superconducting magnets). Indeed, the weak residual Earth field of $2 \mathrm{mG}$ becomes smaller than the lower critical field $H_{c 1}[G] \cong 1700\left(1-T / T_{c}\right)$ of $\mathrm{Nb}$ upon cooling a cavity by only $10^{-5} \mathrm{~K}$ below $T_{c}$. In this case local surface variations of $T_{c}$ (typically much greater than $10^{-5} \mathrm{~K}$ ) result in spotlike penetration and exit of vortices, which become metastable at $T<9 \mathrm{~K}$ and tend to escape the cavity wall. The vortex exit and the complete restoration of the Meissner state at low $T$ is hindered by weak pinning of vortices by crystalline defects. As a result, some fraction of vortices parallel to the surface in the

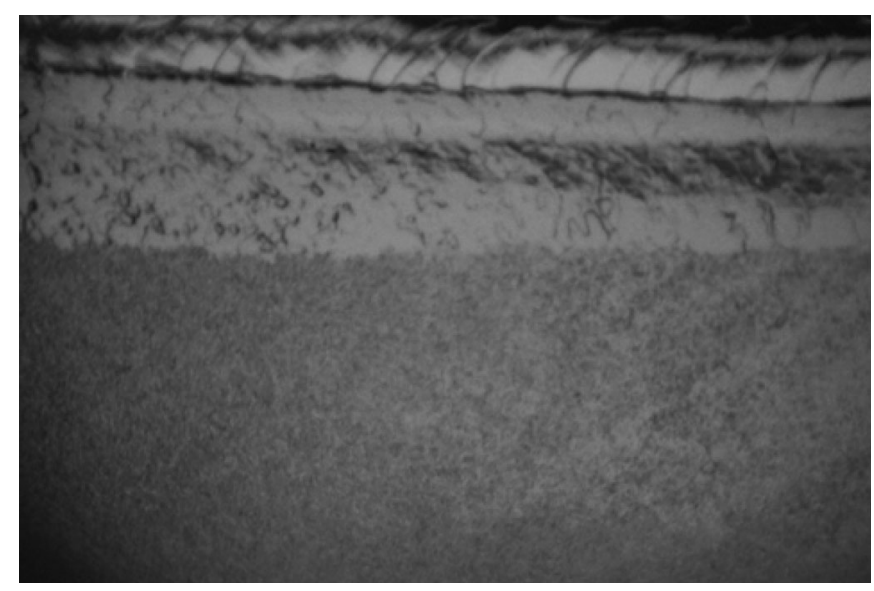

FIG. 20. Image of the inner cavity surface in the equator area at $200^{\circ}$, where high losses were found by temperature mapping. Part of the equator weld is visible at the top of the picture and the heat affected zone ( $\sim 3 \mathrm{~mm}$ wide) with larger grains is underneath. The surface has a general rough appearance and no significant difference was observed at other hot spot locations such as at $250^{\circ}$ or $0^{\circ}$. 
$80 \mathrm{~nm}$ layer of field penetration overcomes weak pinning and escapes upon cooling the cavity down to $2 \mathrm{~K}$, but vortex segments perpendicular to the surface or parallel segments trapped by randomly distributed pinning centers may form complex meandering curves or loops, as discussed in [6]. However, only vortex segments trapped within the rf penetration depth $(<80 \mathrm{~nm})$ can cause significant rf hot spots, whose lateral distribution therefore depends on the distribution of pinning centers which can vary significantly from one cavity to another. Understanding the details of vortex trapping affected by the way the cavity was cooled under weak magnetic fields is beyond the scope of this paper. It was shown, for example, that higher trapping efficiency is achieved if the external field is normal to the cavity surface [15]. Nevertheless, since the diameter of the vortex line $(\approx 80 \mathrm{~nm})$ is much smaller than the wall thickness $(3 \mathrm{~mm})$, the pinned vortex segments may not be necessarily normal to the surface but follow more intricate paths determined by random distribution of pinning centers through the wall thickness. The resulting distribution of pinned vortex bundles can be highly inhomogeneous, which manifests itself in hot spots on the cavity surface.

The lack of improvement of the global surface resistance at high field, as obtained by rf measurements, could be due to the following reasons: (i) a small number of heaters glued on the cavity affects only a small fraction of the cavity surface. (ii) The thermal gradient caused by the heaters on the outer cavity surface tends to push vortices towards the rf surface and along the cavity surface. (iii) The magnitude of $|\nabla T|$, which can be achieved by the heaters used in our experiments may mostly redistribute vortex segments among different pinning sites but be only marginally sufficient to push a significant fraction of vortices out of the sample. The reduction of the intensity of some hot spots would then be due to the breakdown of pinned vortex bundles into vortices being more largely spaced after applying the thermal gradient. In this case the integral losses do not change much, but they are distributed more uniformly over the cavity surface.

Furthermore, additional sources of hot spots cannot be excluded: for example, vortex penetration due to strong rf field at regions of reduced surface barrier may occur, which would not be altered by the application of the thermal gradient. A theoretical description of this phenomenon is provided in Ref. [6], and recent magnetization measurements on niobium samples, which underwent similar treatments as rf cavities, showed a magnetic field of first flux penetration which correlates well with the typical onset field of the $Q$ drop [16].

Grain boundaries in niobium are well-known sites for flux trapping, and some of them were visible in hot spot regions of the large-grain cavity. The results on the finegrain cavity, showing no significant change of temperature maps by local heating, can be explained by the fact that the pinning force increases [higher $J_{c}$ in Eq. (1)] with smaller grain size [7,17], so the thermal gradients produced in our experiments may not be sufficient to depin vortices. A better estimate of $|\nabla T|$ relies on the precise knowledge of the local $J_{c}$, which depends on the nature of pinning centers. On the other hand, the global $J_{c}$ can be inferred from standard transport measurements of voltage-current characteristics on small samples extracted from the $\mathrm{Nb}$ cavity. In addition, since we operated our heaters at $2.0 \mathrm{~K}$, rather than $4.2 \mathrm{~K}$, the thermal gradient we applied was in fact smaller than the estimate given in Sec. I. The reduction of the hot spot intensity by the low-temperature $\left(120^{\circ} \mathrm{C}\right)$ baking can be explained by a reduced flux trapping efficiency near the surface. In fact, in a study of flux trapping at a grain boundary in niobium, it was concluded that the pinning was caused most likely by the presence of impurities segregated at the boundary, rather than by the anisotropy of $H_{c 2}$ or by dislocations at the grain boundary [18]. These impurities could diffuse during bakeout, reducing the pinning efficiency. The nature of these impurities is, at this point, still unclear but the ones that have the highest concentration in niobium, such as hydrogen, oxygen, carbon, and nitrogen, are obvious candidates. Since the $Q$ drop is observed also in single-crystal cavities [19] and the location of hot spots does not always correlate with the presence of a grain boundary [2,20], other pinning mechanisms in niobium must be present.

The $Q$ drop at high field in the $Q_{0}\left(B_{p}\right)$ curves reported in Secs. IV and V appears to be more gradual in the fine-grain cavity, while it seems sharper in the large-grain one. This different behavior had been observed several times in cavities made of the two types of materials, tested at Jefferson Lab. Using the model described in [3], it is possible to gain some insight on this behavior: assuming that, besides the heat dissipated in the uniform part of the cavity, additional power is dissipated at a number of hot spots, the following parametric dependence $Q_{0}\left(B_{p}\right)$ was obtained from a solution of the heat balance equation:

$$
\begin{aligned}
\frac{2 B_{p}^{2}}{B_{b 0}^{2}} & =1+g+u-\sqrt{(1+g+u)^{2}-4 u} \\
Q_{0} & =\frac{Q_{0}(0) e^{-\theta}}{1+g /\left[1-\left(B_{p} / B_{b 0}\right)^{2}\right]}
\end{aligned}
$$

where $\theta=\left(T_{s}-T_{b}\right) \Delta / k T_{b}^{2}, u=\theta e^{1-\theta}, g=\langle\eta\rangle \pi N_{h} L_{h}^{2}$ and $B_{b 0}$ is the breakdown field of the uniform portion of the cavity. $\langle\eta\rangle$ is the average of the parameter $\eta$ which quantifies the extra power generated by a hot spot, $N_{h}$ is the number of hot spots per unit area, and $L_{h}$ is the lateral thermal diffusion length. Equation (3) provides a very good fit of the $Q_{0}\left(B_{p}\right)$ curve of both large-grain and fine-grain cavity, as shown in Fig. 21, with the following value of the fit parameters: $Q_{0}(0)=4 \times 10^{10}, B_{b 0}=110 \mathrm{mT}, g=$ 0.45 for the fine-grain cavity and $Q_{0}(0)=1.8 \times 10^{10}$, 


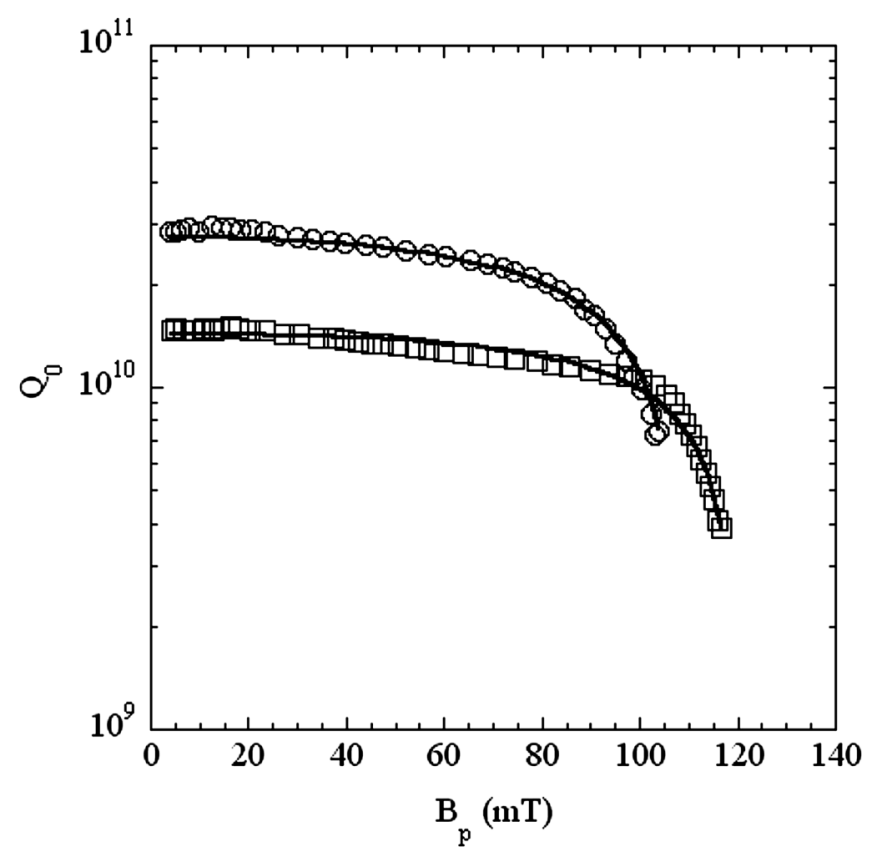

FIG. 21. $Q_{0}\left(B_{p}\right)$ data at $1.7 \mathrm{~K}$ for the fine-grain (circles) and large-grain (squares) cavities fitted with Eq. (3) (solid lines). The data were also shown in Figs. 13 and 17.

$B_{b 0}=121 \mathrm{mT}, g=0.25$ for the large-grain one. The higher $g$ value in the fine-grain cavity than in the largegrain one by almost a factor of 2 may result from a higher number of hot spot sources, possibly due to a larger number of vortices trapped by the denser network of grain boundaries. This explanation would be consistent with the stronger pinning efficiency of the fine-grain structure.

In conclusion, the results in this work show that some of the hot spots observed by thermal maps result from trapped vortices, which reduce the rf performance of superconducting niobium cavities, both at low and high fields. Devising better ways of pushing vortices towards the cavity irises or away from the rf surface using a local thermal gradient ("thermal broom") could therefore be a cheap and effective way to improve the cavity performance. While our experiments enabled us to reveal the underlying physics of vortex hot spots, the local heaters on the outer cavity surface are hardly the most effective and practical means of the trapped flux management in superconducting cavities. Indeed, besides the lateral redistribution of vortex hot spots, such heaters tend to push parallel vortex segments toward the rf surface while not affecting the rf dissipation caused by oscillating vortex segments perpendicular to the cavity surface [6]. In the future, we plan to apply a stronger outward thermal gradient by using a scanning laser beam onto the flat surface of a half-cell niobium cavity, operating in the $\mathrm{TE}_{011}$ mode. Such experiments could further elucidate the mechanisms of dispersing trapped vortex hot spots and reveal the true extent to which rf losses in superconducting cavities can be reduced by applying the "thermal broom" method. A similar experimental setup had been already used at Jefferson Lab to measure the quantum efficiency of a lead cathode deposited on a half-cell cavity [21].

\section{ACKNOWLEDGMENTS}

We would like to thank M. Morrone for the assembly/ disassembly of the temperature mapping system and the heaters, C. Crawford and R. Geng for helping with the Questar telescope, and P. Kushnick for cryogenic support. This manuscript has been authored by Jefferson Science Associates, LLC, under U.S. DOE Contract No. DE-AC0506OR23177.

[1] B. Visentin, in Proceedings of the 11th Workshop on RF Superconductivity, Travemünde, 2003, edited by D. Proch (DESY, Hamburg, Germany, 2004), paper TuO01; G. Ciovati, Physica (Amsterdam) 441C, 44 (2006).

[2] G. Ciovati, P. Kneisel, and A. Gurevich, Phys. Rev. ST Accel. Beams 10, 062002 (2007).

[3] A. Gurevich, Physica (Amsterdam) 441C, 38 (2006).

[4] A. Gurevich, in Proceedings of the 13th Workshop on RF Superconductivity, Beijing, China, 2007, paper TU104, available from http://www.pku.edu.cn/academic/srf2007/ program.html.

[5] G. Arnold-Meyer and W. Weingarten, IEEE Trans. Magn. 23, 1620 (1987); C. Vallet, M. Bolore, B. Bonin, J. P. Charrier, B. Daillant, J. Gratadour, F. Koechlin, and H. Safa, in Proceedings of the 1992 European Particle Accelerator Conference, Berlin, Germany, 1992, edited by $\mathrm{H}$. Henke (Technical University of Berlin, Berlin, 1992), p. 1295.

[6] A. Gurevich and G. Ciovati, Phys. Rev. B 77, 104501 (2008).

[7] A. M. Campbell and J.E. Evetts, Adv. Phys. 21, 199 (1972).

[8] J. Knobloch, H. Muller, and H. Padamsee, Rev. Sci. Instrum. 65, 3521 (1994); G. Ciovati, Ph.D. thesis, Old Dominion University, 2005.

[9] S. W. Van Sciver, Helium Cryogenics (Plenum Press, New York, 1986), Chap. 6.

[10] Available from http://www.ansys.com/.

[11] S. W. Van Sciver, Helium Cryogenics (Plenum Press, New York, 1986), p. 187.

[12] R. M. Sundelin, IEEE Trans. Nucl. Sci. 32, 3570 (1985).

[13] G. Eremeev, H. Padamsee, M. Liepe, and R. Roy, in Proceedings of the 11th Workshop on RF Superconductivity, Travemünde, 2003, Ref. [1], paper MoP18; B. Visentin, J. P. Charrier, Y. Gasser, and S. Regnaud, in Proceedings of the 10th European Particle Accelerator Conference, Edinburgh, Scotland, 2006, edited by C. Biscari, H. Owen, Ch. Petit-Jean-Genaz, J. Poole, and J. Thomason (EPS-AG, Edinburgh, Scotland, 2006), p. 381.

[14] G. Ciovati and A. Gurevich, in Proceedings of the 13th Workshop on RF Superconductivity, Beijing, China, 2007, paper TUP13, available from http://www.pku.edu.cn/ academic/srf2007/proceeding.html. 
[15] Ph. Bernard, G. Cavallari, E. Chiaveri, E. Haebel, H. Lengeler, H. Padamsee, V. Picciarelli, D. Proch, A. Schwettman, J. Tuckmantel, and W. Weingerten, Nucl. Instrum. Methods 206, 47 (1983).

[16] S. B. Roy, G. R. Myneni, and V. C. Sahni, Phys. Rev. ST Accel. Beams (to be published).

[17] A. T. Santhanam, J. Mater. Sci. 11, 1099 (1976).

[18] A. DasGupta, C. C. Koch, and D. M. Kroeger, Philos. Mag. 38, 367 (1978).

[19] P. Kneisel, G. Ciovati, W. Singer, X. Singer, D. Reschke, and A. Brinkmann, in Proceedings of the 11th European Particle Accelerator Conference, Genoa, 2008 (EPS-AG,
Genoa, Italy, 2008), paper MOOP136, available from http://accelconf.web.cern.ch/accelconf/.

[20] G. Eremeev and H. Padamsee, in Proceedings of the 10th European Particle Accelerator Conference, Edinburgh, Scotland, 2006, Ref. [13], p. 475.

[21] J. Smedley, T. Rao, P. Kneisel, J. Sekutowicz, J. Iversen, D. Klinke, D. Kostin, W. Möller, A. Muhs, R. Lefferts, and A. Lipski, in Proceedings of the 2007 Particle Accelerator Conference, Albuquerque, New Mexico, 2007, edited by S. Schriber (IEEE, Albuquerque, New Mexico, 2007), p. 1365 . 